\title{
Towards Understanding Prices and Ethics: Ethical Consumers with Price-Dependent Utilities
}

\author{
Pascal Stiefenhofer \\ Newcastle University Business School, Newcastle upon Tyne, UK \\ Email: pascal.stiefenhofer@newcaslte.ac.uk
}

How to cite this paper: Stiefenhofer, P. (2021). Towards Understanding Prices and Ethics: Ethical Consumers with Price-Dependent Utilities. Theoretical Economics Letters, 11, 477-484. https://doi.org/10.4236/tel.2021.113031

Received: April 20, 2021

Accepted: June 1, 2021

Published: June 4, 2021

Copyright $\odot 2021$ by author(s) and Scientific Research Publishing Inc. This work is licensed under the Creative Commons Attribution International License (CC BY 4.0).

http://creativecommons.org/licenses/by/4.0/

\section{(c) (i) Open Access}

\begin{abstract}
This paper analysis ethical consumption behavior with price-dependent ethical preferences. It extends the definition of goods to include ethical values in the preferences and introduces a mathematically tractable definition of social labels paving the way for new theoretical and empirical developments. Relationships between the standard economic model and the ethical consumption model with price-dependent utilities are established.
\end{abstract}

\section{Keywords}

Ethical Consumption, Price-Dependent Utility, Ethical Prices

\section{Introduction}

The theory on price-dependent preferences is sparse. The various hypotheses motivating the study of price-dependent preferences are: judging the quality of a good by its price (Veblen, 1899), "Snob appeal" of a good (Scitovsky, 1945), and treatment of money and other financial assets in temporal equilibrium models (Samuelson, 1947). The bulk of this research predominantly focuses on motivating price-dependent preferences and deriving generalizations and variations of the Slutsky equation with their implications for welfare analysis (Kalman, 1968; Pollak, 1977; Arrow \& Hahn, 1991).

In this paper, we consider a new hypothesis demanding price-dependent preferences. In our model, prices do not only perform the role of efficient resource allocations but also inform consumers about ethical attributes of production and distribution. An increasing number of consumers base their daily consumption decisions on ethical values, such as human rights, environmentally friendly pro- 
duction, sustainable production and distribution standards, animal well-being, and fair prices ${ }^{1}$. Ethical consumers, hence, are consumers who beyond making rational decisions on price-quantity apply certain values when making consumption decisions. Ethical consumers feel responsible towards society and express these feelings via their purchasing behavior ${ }^{2}$. Examples of positive ethical consumption include the consumption of fair trade, organic, and green products. Ethical consumers boycotting the consumption of unethically produced and distributed commodities and services exhibit negative consumption. This peculiar consumption behavior is unique to ethical consumers.

Motivated by the hypothesis that some consumers like to display their ethical consumption choices, this paper extends the theory of price-dependent utilities to ethical consumption introduced in (Stiefenhofer, 2019; Stiefenhofer \& Zhang, 2020; Stiefenhofer, 2021) by providing a mathematically tractable definition of a voluntary social label, which characterizes the ethical consumption space, and therewith, facilitates new empirical and theoretical developments. It is shown that the ethical consumption model can be reduced to the standard economic model for the special class of log-linear utility functions. Section two introduces the model and new definitions. Section three discusses the main results followed by a conclusion.

\section{The Ethical Consumption Model}

We assume there exists a group of socially responsible consumers represented by an index $i=1, \cdots, m$ who do not only care about their own consumption of goods $l=1, \cdots, n$, with consumption bundle $x_{i} \in \mathbb{R}_{+}^{n}$, but they also care about certain ethical and social conditions $j=1, \cdots, k$ regarding production and distribution of these goods. Associated with a consumption bundle there is a price system $p \in \mathbb{R}_{+}^{n}$. Let the ethical consumer price $\mathfrak{p}$ be defined by the global market price $p_{M}$ plus a premium $p_{E}$ such that

$$
\mathfrak{p}=p_{M}+p_{E} \geq p_{\min },
$$

where $p_{\min }>0$ and $P_{E} \geq 0$ is a premium (Dragusanu \& Nunn, 2014; Figure 1 on pp. 219-220) and $\mathfrak{p}=\left(\mathfrak{p}^{1}, \cdots, \mathfrak{p}^{n}\right) \in \mathbb{R}_{++}^{n}$ satisfying $\mathcal{S}:=\left\{\mathfrak{p} \in \mathbb{R}_{++}^{n}: \mathfrak{p}^{n}=1\right\}$. Relative prices lead to demand functions which are homogeneous of degree zero. Such functions provide meaningful welfare analysis (Pollak, 1977; Arrow \& Hahn, 1991). In our model prices do not only signal the scarcity of resources in their efficient allocation process but also all these things our consumers care about regarding ethical production and distribution.

Associated with an ethical price system $\mathfrak{p}$ there are $l=1, \cdots, n$ ethically produced and distributed consumption goods. We now characterize a consumption good $x_{i}^{l} \in \mathbb{R}_{+}$in terms of ethical values such as no child labor, environmental

${ }^{1}$ Ethical consumption is defined in some detail in Tallontire, A. and Blowfield, M. (2001), Ethical consumers and ethical trade; A review of the current literature. Policy Series 12, pp. 1-35.

${ }^{2}$ Doane, D. (2001), Taking fight: The rapid growth of ethical consumerism, New Economics Foundation. 
friendly production, minimum wages, safe work conditions, gender equality, human rights, etc. While some of these ethical values are quantifiable, others are clearly not. Hence, we introduce an information array $e^{l}$ collecting the words and symbols about production conditions associated with a good $x_{i}^{l}$. We assume that each good $l$ is characterized by $j=1, \cdots, k$ ethical values $e^{l j}=\left(e^{l 1}, \cdots, e^{l k}\right)^{3}$. Each ethical value is indexed by $j \in 1, \cdots, k$ and is assumed to be known to an informed consumer. The collection of all ethical values associated with a consumption bundle $x_{i}$ is an information array given by

$$
e=\left(\begin{array}{ccc}
e^{11} & \ldots & e^{1 k} \\
\vdots & \ddots & \vdots \\
e^{n 1} & \ldots & e^{n k}
\end{array}\right) .
$$

It follows that a consumption good is characterized by a quantifiable physical property $x_{i}^{l}$ and an associated set of ethical values $e^{l}$, providing a description of production and distribution conditions regarding environment, health, gender, animal well-being, etc.. An ethically produced good is hence denoted by $\left.x_{i}^{l}\right|_{e^{l}} \in \mathbb{R}_{++}$. Note that $\left.x^{l}\right|_{e^{l}} \neq\left. x^{l}\right|_{e^{l^{\prime}}}$ for $e^{l} \neq e^{l^{\prime}}$. Our characterization of goods states that two physically identical goods produced under different ethical conditions are "nearly-identical" goods. The physically identical goods only differ in the way they are produced. Ethical consumers are informed consumers who can distinguish between such goods. A social label conveys this information to consumers. Our definition of a consumption good is a generalization of the characterization of goods by (Debreu, 1959), which are defined by their physical, temporal, and spatial nature, to goods which also depend on a set of ethical values. We denote an ethical consumption bundle $\left.x_{i}\right|_{e}$ by

$$
\chi_{i} \in \mathcal{X}_{i}
$$

where $\mathcal{X}_{i} \subseteq \mathbb{R}_{+}^{n}$ is the consumption space characterized by ethical attributese. We label goods $\chi_{i} \in \mathcal{X}_{i}$ satisfying such characteristics as ethical conspicuous goods. We formalize this idea in the subsequent three definitions.

Definition 1 (Ethical conspicuous goods). An ethical conspicuous consumption bundle $\chi_{i} \in \mathcal{X}_{i}$ is defined by a consumption bundle $x_{i} \in \mathbb{R}_{+}^{n}$ characterized by an array of descriptive ethical values $e$.

Using (1) and (2) we introduce a definition of a voluntary social label that is based on a price signal $\mathfrak{p}\left(p_{M}, p_{E}\right)$ which informs consumers $i=1, \cdots, m$ about ethical production and distribution processes, and a class of ethically characterized commodities $\chi_{i} \in \mathcal{X}_{i}$, which further informs consumers descriptively about the way goods are produced.

Definition 2 (Voluntary social label). A voluntary social label $\mathfrak{S}$ is defined by a pair $\left(\mathfrak{p}, \chi_{i}\right)$ consisting of an ethical price system $\mathfrak{p} \in \mathcal{S}$ and commodity bundle $x_{i} \in \mathbb{R}^{n}$ characterized by a set of ethical standards e represented in words

${ }^{3}$ Note that $e^{l}$ is not a quantity in $\mathbb{R}^{k}$ as in the Lancaster (1971) model, but a descriptive list of characteristics. Hence, it should not be confused with the mathematical definition of a vector, i.e., the inner product operation does not apply. In essence $e^{l}$ is a string of information. 
and symbols, which, is intended to inform consumers about ethical production and distribution standards. A consumer $i=1, \cdots, m$ who's consumption choices are informed by a social label $\mathfrak{S}$, i.e.,

$$
\mathfrak{S}_{i}:=\left(\mathfrak{p}, \chi_{i}\right) \in \mathcal{S} \times \mathcal{X}_{i}
$$

belongs to the class of ethical consumers.

A voluntary social label $\mathfrak{S}_{i}$ consists of an ethical price and ethical conspicuous commodity pair $\left(\mathfrak{p}, \chi_{i}\right)$ which belongs to the set $\mathcal{S} \times \mathcal{X}_{i}$. Definition 2 allows us to consider a group of informed consumers, where $\mathfrak{S}_{i}$ represents that $\mathfrak{S}$ is known to $i=1, \cdots, m$. These consumers have ethical preferences in the sense that they care about the way goods are produced and distributed. Consumers satisfying definition 2 are classified as ethical consumers. It remains to characterize their consumption behavior.

Definition 3 (Conspicuous ethics). Conspicuous ethics refers to an ethical consumption behavior where an informed consumer $\mathfrak{S}_{i}$, for $i=1, \cdots, n$, expresses superior ethical responsibility towards society relative to other members of society by purchasing ethically produced and distributed goods $\chi_{i}$ at ethical prices $\mathfrak{p}$. It is assumed that ethical consumers derive esteem from displaying their social responsibility through the consumption of ethically conspicuous goods. Positive ethical consumption is satisfied for all pairs

$$
\left(\mathfrak{p}, \chi_{i}\right) \in \mathfrak{S}_{i} .
$$

An ethical consumer also expresses superior ethical responsibility towards society by boycotting the consumption of unethically produced and distributed goods. Negative ethical consumption is satisfied if

$$
\text { either } p \neq \mathfrak{p} \text { or } x_{i} \neq \chi_{i} \text {. }
$$

Ethical consumers base their consumption decisions on the basis of ethical values, such as human rights, environmentally friendly and sustainable production, and animal well-being. We hypothesize that they express moral superiority towards society via positive and negative consumption of ethical conspicuous goods. We consider ethical consumers who derive esteem from the consumption of "more expensive" ethical conspicuous goods. This relates our model to Veblen (1899) who discusses the evolution of society based on the competition of esteem and price-dependent preferences. This is similar to our model, the motivation for priced dependent preferences, however, is different.

We characterize the consumer's preference ordering $\succeq_{q}$ by an ethical price index $q$ associated with an ethical market price $\mathfrak{p}$. It is assumed that the price depending preference ordering $\succeq_{q}$ is unaffected by a proportional change in all prices $\mathfrak{p}$ (Pollak, 1977). Moreover, we consider normalized prices $q$, hence, our characterization of the consumer preference is at variance to Kalman, who assumes a preference ordering of the form $\succeq_{p}$ over a consumption-market price space $(x, p) \in \mathbb{R}_{+}^{n} \times \mathbb{R}_{++}^{n}$, where $x$ is a commodity bundle (Kalman, 1968: A.1, pp. 498). Stiefenhofer and Zhang (2020) consider a class of price-dependent ethical 
utility functions.

We want to represent preferences, which do not only depend on ethical consumption bundles but also on relative prices, which inform consumers about the way goods are produced. We are going to consider utility functions of the form $u_{i}\left(\chi_{i}, q\right)$. Let the open normalized price simplex be denoted by

$$
\mathbb{S}:=\left\{\mathfrak{p} \in \mathbb{R}_{++}^{n}: \sum_{k=1}^{n} \mathfrak{p}^{k}=1\right\},
$$

with its closure simplex defined by

$$
\overline{\mathbb{S}}:=\left\{\mathfrak{p} \in \mathbb{R}_{+}^{n}: \sum_{k=1}^{n} \mathfrak{p}^{k}=1\right\}
$$

We now formalize the hypothesis that consumers do not only have preferences for the physical properties of goods, but they also care about the way goods are produced. Hence by (2) and (4) we defined a preference relation of the form $\succeq_{(q, \chi)}$ over the preference ordering space $\left(q, \chi_{i}\right) \in \overline{\mathbb{S}} \times \mathcal{X}_{i}$, where

$$
u_{i}: \overline{\mathbb{S}} \times \mathcal{X}_{i} \rightarrow \mathbb{R}
$$

is the price-dependent ethical utility function. Stiefenhofer (2019) establishes the geometric conditions of smooth ethical utility functions. Stiefenhofer and Zhang (2020) provide an example of a price-dependent ethical utility function. We can now formulate an ethical consumption model, where production is implicitly formulated through a reallocation of initial endowments. Let an ethical consumer $i=1, \cdots, m$ be endowed with a vector of initial endowments

$\left.\omega_{i}\right|_{e}=\left.\left(\left.\omega_{i}^{1}\right|_{e^{1}}, \cdots,\left.\omega_{i}^{n}\right|_{e^{n}}\right) \in \Omega_{i}\right|_{e} \subseteq \mathbb{R}_{++}^{n}$. We denote $\left.\omega_{i}\right|_{e}$ by $\omega_{i}$ and $\left.\Omega_{i}\right|_{e}$ by $\Omega_{i}{ }^{4}$.

\section{Main Results}

Model I: Consider a group of informed consumers $\mathfrak{S}_{i}$, for $i=1, \cdots, m$ satisfying definition 2 . Let an ethical consumer $i=1, \cdots, m$ be endowed with a vector of initial endowments $\omega_{i} \in \Omega_{i}$, and let his set of feasible consumption allocations be defined by $\mathcal{B}_{i}\left(\mathfrak{p}, \omega_{i}\right):=\left\{\left(\mathfrak{p}, \chi_{i}\right) \in \mathcal{S} \times \mathcal{X}_{i}: \mathfrak{p} \cdot \chi_{i} \leq \mathfrak{p} \cdot \omega_{i}\right\}$. Then for any given relative price system $q \in \mathbb{S}$ (ethical price index), ethical market price system $\mathfrak{p} \in \mathcal{S}$, and ethical conspicuous goods $\chi_{i} \in \mathfrak{S}_{i}$ satisfying definition 1 the objective of the consumer is defined by

$$
\underset{\chi_{i} \in \mathcal{B}_{i}\left(\mathfrak{p}, \omega_{i}\right)}{\arg \max } u_{i}\left(\chi_{i} ; q\right)
$$

for every $i=1,2, \cdots, m$. By definitions 1 and 3 ethical consumers consider the pair $\left(\mathfrak{p}, \chi_{i}\right) \in \mathfrak{S}_{i}$ in their consumption decision. Other consumption bundles are boycotted, since they do not belong to the set of ethically produced goods. In addition consumption must also satisfy the wealth constraint $\chi_{i} \in \mathcal{B}_{i}\left(\mathfrak{p}, \omega_{i}\right)$.

${ }^{4}$ We do not intend to explicitly model ethical production in this paper. We are, however, aware that this is another interesting problem. 
The disentanglement of the price system into a price index $q$ which enters the utility function and a price system $\mathfrak{p}$ which enters the budget constraint is a distinct property of our model ${ }^{5}$.

This model captures two key elements of ethical consumption. 1) Consumption is restricted to the consumption of ethically produced goods as indicated by the social label $\mathfrak{S}_{i}$, and 2) the utility of the consumer depends on some price index $q$, which, further informs the consumer about ethically produced goods. Model I provided by (6) is obviously a stylized model and the relation between the ethical price system $\mathfrak{p}$ and the price index $q$ needs to be established in more detail since the observed market price $\mathfrak{p}$ informs the consumer. Nevertheless, it allows us to show the following result:

Theorem 1. Consider the class of log-linear ethical utility functions. Then

$$
\underset{\chi_{i} \in \mathcal{B}_{i}\left(\mathfrak{p},\left.\omega_{i}\right|_{e}\right)}{\arg \max } u_{i}\left(\chi_{i} ; q\right) \Leftrightarrow \underset{x_{i} \in \mathcal{B}\left(p, \omega_{i}\right)}{\arg \max } u_{i}\left(x_{i}\right) .
$$

Proof. Let $x_{i}=\chi_{i}$ be the same commodity bundle for $x_{i} \in \mathbb{X}_{i} \subseteq \mathbb{R}_{++}^{n}$. Then $\left.x_{i} \in \Omega_{i}\right|_{e}$. Let $u\left(x_{i}\right)$ be a Cobb-Douglas function with parameters $\alpha_{l}>0$ and $\sum_{l=1}^{n} \alpha_{l}=1$. It is well known that for any $\left(p, \omega_{i}\right) \in \mathcal{S} \times \mathbb{R}_{++}$, where $w_{i} \in \mathbb{R}_{++}$is the consumer's wealth as defined by $p \cdot \omega_{i}$ with $\left.\omega_{i} \in \Omega_{i}\right|_{e}$, this problem has a unique solution $\phi_{i}\left(p, w_{i}\right)$ under the usual strict concavity assumptions of $u_{i}($.$) . Its map is given by$

$$
\phi_{i}: \mathcal{S} \times \mathbb{R}_{++} \rightarrow \mathbb{R}_{++}^{n} \text {, }
$$

which is homogeneous of degree zero. Now, with the price vector $q \in \mathbb{S}$ being kept fixed, and by defining $q_{l}=\alpha_{l}$ for $l=1, \cdots, n$ such that $q_{l}>0$ and $\sum_{l=1}^{n} q_{l}=1$ it follows, by the usual arguments, that the consumer's maximization problem has a unique solution $\xi_{i}\left(q, p, w_{i}\right)$ for any $\left(p, \omega_{i}\right) \in \mathcal{S} \times \mathbb{R}_{++}$and $q \in \mathbb{S}$, which is called the extended demand function of the standard demand function. Its map is given by

$$
\xi_{i}=\mathbb{S} \times\left(\mathcal{S} \times \mathbb{R}_{++}\right) \rightarrow \mathbb{R}_{++}^{n},
$$

which is homogeneous of degree zero. Moreover, it can easily be shown that for the class of utility functions considered here, the price-dependent utility function $u(. ; q)$ is homogeneous of degree zero.

Theorem 1 states the conditions of how we can relate the ethical consumption model to the standard consumer model and therewith suggesting that parameters entering the utility of the ethical consumer can easily be estimated empirically. Next, we endogenize the disentangled price system.

Definition 4. The disentanglement of the ethical commodity market price $\mathfrak{p}$ into a price index $\mathrm{q}$ is given by a map

$$
s: \mathcal{S} \rightarrow \mathbb{S}
$$

${ }^{5}$ The semicolon in the utility function indicates that the price index $q$ is considered as a parameter. This is very desirable because it can be estimated empirically. The parameter $q$ characterizes how an indifference map is placed into the consumption space. 
defined by $s(\mathfrak{p}):=\frac{\mathfrak{p}^{k}}{\sum_{k=1}^{n} \mathfrak{p}^{k}}$ for $k=1, \cdots, n$.

We now consider a characterization of the consumer's preferences for ethically produced goods based on the market price $\mathfrak{p}$. Using definitions 1,3 and 4 we extend our basic model of ethical consumption to obtain:

Model II: Consider a group of informed consumers $\mathfrak{S}_{i}$, for $i=1, \cdots, m$ satisfying definition 2 . Let an ethical consumer $i=1, \cdots, m$ be endowed with a vector of initial endowments $\omega_{i} \in \Omega_{i}$, and let his set of feasible consumption allocations be defined by $\mathcal{B}_{i}\left(\mathfrak{p}, \omega_{i}\right):=\left\{\left(\mathfrak{p}, \chi_{i}\right) \in \mathcal{S} \times \mathcal{X}_{i}: \mathfrak{p} \cdot \chi_{i} \leq \mathfrak{p} \cdot \omega_{i}\right\}$. Then for any given relative price system $q \in \mathbb{S}$ satisfying definition 4 (ethical price index), ethical market price system $\mathfrak{p} \in \mathcal{S}$, and ethical conspicuous goods $\chi_{i} \in \mathfrak{S}_{i}$ satisfying definition 1 the objective of the consumer is defined by

$$
\underset{\chi_{i} \in \mathcal{B}_{i}\left(\mathfrak{p}, \omega_{i}\right)}{\arg \max } u_{i}\left(\chi_{i} ; s(\mathfrak{p})\right) \text {. }
$$

Theorem 2. Consider the class of log-linear ethical utility functions. Then

$$
\underset{\chi_{i} \in \mathcal{B}_{i}\left(\mathfrak{p}, \omega_{i}\right)}{\arg \max } u_{i}\left(\chi_{i} ; q\right) \Leftrightarrow \underset{\chi_{i} \in \mathcal{B}_{i}\left(\mathfrak{p}, \omega_{i}\right)}{\arg \max } u_{i}\left(\chi_{i} ; s(\mathfrak{p})\right) .
$$

Proof. Recall that $u_{i}\left(\chi_{i} ; q\right)$ is homogeneous of degree zero. Then, using definition (4) it follows that $u_{i}\left(\chi_{i} ; s(\mathfrak{p})\right)$ is also homogeneous of degree zero. Note that the extended demand mapping

$$
\xi_{i}=\mathbb{S} \times\left(\mathcal{S} \times \mathbb{R}_{++}\right) \rightarrow \mathbb{R}_{++}^{n}
$$

reduces to

$$
\phi_{i}: \mathcal{S} \times \mathbb{R}_{++} \rightarrow \mathbb{R}_{++}^{n},
$$

which turns out to be the same demand function as the standard consumer model.

From theorems 1 and 2 we obtain the following corollary.

Corollary 1.

$$
\underset{x_{i} \in \mathcal{B}\left(p, \omega_{i}\right)}{\arg \max } u_{i}\left(x_{i}\right) \Leftrightarrow \underset{\chi_{i} \in \mathcal{B}_{i}\left(\mathfrak{p}, \omega_{i}\right)}{\arg \max } u_{i}\left(\chi_{i} ; q\right) \Leftrightarrow \underset{\chi_{i} \in \mathcal{B}_{i}\left(\mathfrak{p}, \omega_{i}\right)}{\arg \max } u_{i}\left(\chi_{i} ; s(\mathfrak{p})\right) .
$$

\section{Conclusion}

Empirical studies suggest that there is a group of consumers with a high willingness to pay for ethically certified goods (Pelsmacker \& Rayp, 2005). In our model, we explain this phenomenon by hypothesizing that ethical consumers maximize moral esteem by purchasing the more expensive ethical consumption goods. At the heart of this consumption behavior lies the assumption of conspicuous ethics suggesting a positive correlation between prices and demand for ethically produced goods.

Ethical consumers also care about minimum prices guaranteeing that producers comply with minimum wages and other ethical production standards. Commodities that do not satisfy a minimum price condition and some ethical characterization as described by a social label are boycotted by ethically conscious 
consumers. In our model, the voluntary social label performs the role of separating the positive ethical consumption space from the negative consumption space, where consumers boycott consumption of unethically produced commodities $^{6}$. The Fair Trade label is an example of a voluntary social labeling initiative based on an organized social movement and market-based approach to help producers in developing countries to obtain better trading conditions and promote sustainability. Fair Trade is a project generated by the demand for ethically labeled goods.

A possible venue for future research is to empirically investigate the premises of the ethical consumption model discussed in this paper. Very little empirical research on price-dependent utility functions exists and there are interesting venues to pursue; such include measuring the willingness to pay and the role prices play in informing consumer choices. The latter requires investigating the index function $s(\mathfrak{p})$ introduced in Model II.

\section{Conflicts of Interest}

The author declares no conflicts of interest regarding the publication of this paper.

\section{References}

Arrow, K., \& Hahn, F. (1991) General Competitive Analysis. North Holland: North Holland.

Debreu, G. (1959). Theory of Value: An Axiomatic Analysis of Economic Equilibrium. London: Yale University Press.

Dragusanu, R., \& Nunn, N. (2014). The Economics of Fair Trade. Journal of Economic Perspectives, 28, 217-236. https://doi.org/10.1257/jep.28.3.217

Kalman, P. (1968). Theory of Consumer Behavior When Prices Enter the Utility Function. Econometrica, 36, 497-510. https://doi.org/10.2307/1909519

Pelsmacker, P., \& Rayp, L. (2005). Do Consumers Care About Ethics? The Journal of Consumer Affairs, 39, 363-385. https://doi.org/10.1111/j.1745-6606.2005.00019.x

Pollak, R. (1977). Price-Dependent Preferences. American Economic Review, 67, 64-75.

Samuelson, P. A. (1947). Foundations of Economic Analysis. Cambridge, MA: Harward University Press.

Scitovsky, T. (1945). Some Consequences of the Habit of Judging Quality by Price. Review of Economic Studies, 12, 100-105. https://doi.org/10.2307/2296093

Stiefenhofer, P. (2019). Conspicuous Ethical Consumption. Theoretical Economics Letters, 9, 1-8. https://doi.org/10.4236/tel.2019.91001

Stiefenhofer, P. (2021). Conspicuous Ethics: Existence of Price-Dependent Ethical Utility Functions. Applied Mathematics, 12, 252-261. https://doi.org/10.4236/am.2021.124016

Stiefenhofer, P., \& Zhang, W. (2020). Conspicuous Ethics: A Veblen Effect Condition for Ethical Consumption Goods. Applied Economics Letters, 28, 217-236. https://doi.org/10.1080/13504851.2020.1855306

Veblen, T. (1899). The Theory of the Leisure. New York: Macmillan.

${ }^{6}$ The literature refers to negative consumption as the boycott of consumption. Consumption boycotts are organized by ethical consumer organizations. 